\section{Dr. Vlachoyiannopoulos replies}

\section{To the Editor:}

We read with interest the comments of Drs. Barbhaiya and Erkan ${ }^{1}$ on our work published in The Journal ${ }^{2}$. The authors raised 2 important issues. The first was that as time passes, a great deal of organ damage is accumulated in patients with antiphospholipid syndrome (APS), significantly affecting their quality of life. We all agree with this, whichever way we quantify this damage. For instance, Erkan, et al in a previous report counted in a simple and informative way the events leading to disability ${ }^{3}$, to prove that accumulated damage characterizes the progression of APS. When, however, we have to evaluate large patient cohorts for a long time, a damage index offers a rather generalized view of the disability of the patient populations and helps the policymaking authorities to plan health policies. The second issue is that the Systemic Lupus International Collaborating Clinics (SLICC)/American College of Rheumatology (ACR) Damage Index (DI) ${ }^{4}$ is probably not the optimal tool for the assessment of organ damage in APS because it does not record all the aPL-related damage and also does not define in a proper way damage related to venous thrombosis.

We used the SLICC/ACR DI because it is the only well described, already published and validated tool to measure organ damage for patients with systemic lupus erythematosus (SLE). At the time of the preparation of our manuscript, the abstracts suggested by Drs. Barbhaiya and Erkan ${ }^{5,6}$ had not been published, and to our knowledge these papers are still known in the form of abstracts. We agree that this tool has not been validated for patients with APS; however, the items included in this instrument cover almost completely the clinical events related to multiple brain and peripheral infarcts or to venous thrombotic events. For instance, the neuropsychiatric section of SLICC/ACR DI includes the items cognitive impairment, seizures requiring therapy for 6 months, cerebrovascular accident ever, cranial or peripheral neuropathy (excluding optic), and transverse myelitis. One should realize that the SLICC/ACR instrument was built on clinical grounds, thus items suggested by the authors of this letter such as "multiple sclerosis-like disease," "diffuse pulmonary hemorrhage resulting in chronic symptoms," or "white matter changes" are neither well defined nor at all clinical. We do not know whether the modifications to the SLICC/ACR DI suggested by Drs. Barbhaiya and Erkan ${ }^{5}$ will provide more accurate and meaningful measurements of DI in APS because their work is still unpublished and needs validation. The work of Amigo, et al aiming to validate a new physician-reported chronic DI in APS patients (DIAPS $)^{6}$ is also unpublished. Finally, when we read the EuroQol paper ${ }^{7}$, we realized that even this is a position paper and needs validation.

We hope that the work of Barbhaiya, et $a l^{5}$ and Amigo, et al ${ }^{6}$ will appear soon in the literature in the form of full-length articles and will provide more accurate DI measurements when applied in patients with
APS. There is, however, a theoretical question: because SLE and APS are diseases that overlap and evolve from one to the other, how can anyone be sure that a brain symptom, for instance, is related to demyelinating, to thromboembolic, or to vasculitic process, especially when evaluating the patients' files retrospectively? Although this kind of differentiation is important for understanding pathogenicity and probably of planning therapeutic interventions (we do not have many), it is probably meaningless when evaluating endstage organ damage. Let us invest our efforts in understanding pathogenesis and not in discussing minor things.

PANAYIOTIS G. VLACHOYIANNOPOULOS, MD, Professor of Medicine-Immunology, Department of Pathophysiology, Medical School, National University of Athens, 75 Mikras Asias St., 11527 Athens, Greece.E-mail: pvlah@med.uoa.gr

\section{REFERENCES}

1. Barbahaiya M, Erkan D. The optimal tool for assessment of organ damage in antiphospholipid syndrome. J Rheumatol 2013;40:89.

2. Grika EP, Ziakas PD, Zintzaras E, Moutsopoulos HM, Vlachoyiannopoulos PG. Morbidity, mortality, and organ damage in patients with antiphospholipid syndrome. J Rheumatol 2012;39:516-23.

3. Erkan D, Yazici Y, Sobel R, Lockshin MD. Primary antiphospholipid syndrome: functional outcome after 10 years. J Rheumatol 2000;27:2817-21.

4. Gladman D, Ginzler E, Goldsmith C, Fortin P, Liang M, Urowitz $\mathrm{M}$, et al. The development and initial validation of the Systemic Lupus International Collaborating Clinics/American College of Rheumatology damage index for systemic lupus erythematosus. Arthritis Rheum 1996;39:363-9.

5. Barbhaiya M, Erkan D, Rodriguez-Almaraz E, Ramon G, Vega J, Lockshin MD. Utility of the Systemic Lupus International Collaborating Clinics (SLICC)/American College of Rheumatology (ACR) Damage Index for antiphospholipid antibody (aPL) positive patients [abstract]. Arthritis Rheum 2011;63 Suppl:S3.

6. Amigo M, Barile LA, Barragan A, Espinosa-Cuervo G, Goycochea M, Martinez-Martinez LA, et al. Development and initial validation of a chronic damage index in patients with antiphospholipid syndrome [abstract]. Arthritis Rheum 2011;63 Suppl:S3.

7. EuroQol - A new facility for the measurement of health-related quality of life. The EuroQol Group. Health Policy 1990; 16:199-208.

J Rheumatol 2013;40:1; doi:10.3899/jrheum.120976 